\section{Starting with stars}

\section{John Lattanzio}

The Fundamentals of Stellar Astrophysics. By George W. Collins II. W.H. Freeman: 1989. Pp.494. \$47.95, £37.95.

THE theory of stellar astrophysics is naturally divided into the study of interiors and atmospheres. Collins has provided a convenient introduction to both subjects in the one text, aimed at first-year graduate students or advanced undergraduates. The book contains a thorough index, and provides a list of references and sources for further reading that will act as an excellent point of entry to the more advanced literature.

The text is divided into two parts, the first of which deals with stellar interiors. Unfortunately, the opening chapter contains a great deal of statistical mechanics. Although this material is clearly essential to the development of the subject, it may seem an anti-climax to the student impatient to deal with stars and an appendix would have been a better place for it. Collins gives appropriate attention to scaling arguments to familiarize students with the techniques, and his selection of theorems from Chandrasekhar's An Introduction to the Study of Stellar Structure is excellent. I feel that the section on nuclear energy generation is too brief, though it would serve as an introduction to the definitive treatment to be found in Clayton's Principles of Stellar Evolution and Nucleosynthesis.

Unexpected inclusions are the chapters on relativistic stellar structure and the structure of distorted stars. To my mind, however, the former would have been better omitted in favour of more detail in other sections. The final chapter of this part, which deals with pulsation and oscillation, seems to have been introduced for completeness. Ideally the stellar-evolution chapter would have mentioned variable stars (where they are seen in the Hertzsprung-Russell diagram, and how their evolution puts them there), and this chapter would then have been the necessity that it is.

Moving on to stellar atmospheres, Collins derives the equations of radiative transfer and illustrates various approximations which enable their solution. He then discusses the interaction between the gas and the radiation field. The chapter that should then be the reward for these toils, which is entitled "Construction of a Model Stellar Atmosphere", is somewhat disappointing because no example is provided.

The next two chapters deal with the formation and shape of spectral lines.
Collins then looks at departures from local thermodynamic equilibrium, and concludes with an account of complications which exist in real stars (stellar winds, for example) but which have been ignored in the standard theory.

More diagrams would have been appropriate (the account of stellar evolution, for example, contains but two HR diagrams), as would some example models of stellar interior and atmospheres both as illustrations of the concepts described in the text and to show the student the rewards of learning the physics and doing the calculations. Problems are given at the end of each chapter, and they cover a wide variety of styles. Some require a

\section{Michael Rowan-Robinson}

Encyclopedia of Astronomy and Astrophysics. Edited by Robert A. Meyers and Steven N. Shore. Academic: 1989. Pp.807. \$49.95, £31.50.

THE reader turns to an encyclopaedia when he or she is looking for detailed information about an unfamiliar topic. These days science is so specialized, and its practitioners are forced to be so narrow in their professional interests, that even in a scientist's own field a high proportion of topics will come into that category. So there is every reason why astronomers (and others) need a book such as the Encyclopedia of Astronomy and Astrophysics.

There are two conditions that an encyclopaedia has to satisfy. The first is that the entries must be satisfyingly packed with useful facts and information; the second is that the encyclopaedia must give in total a reasonably comprehensive survey of the area being considered. Many of the entries in the present offering satisfy the first of these requirements, especially where the topic is fairly narrowly defined and is discussed by recognized experts. "Infrared Astronomy" (by Robert Gehrz, Gary Grasdalen and John Hackwell), "Neutrino Astronomy" (Raymond Davis $\mathrm{Jr}$ ), "Stellar Structure and Evolution" (Peter Bodenheimer) and "Supernovae" (David Branch), to pick just a few, are both interesting and authoritative.

The same cannot be said of the many entries supplied by one of the editors and those dealing with topics that are just too broad (astrophysics and cosmology, for example). These just read like fillers and do not provide enough information in the form that an encyclopaedia-reader seeks. Perhaps this compilation reveals its genesis a bit too readily. About half of the stellar interior code or an atmospheres code for their completion! It is a pity that there is no section on the growing field of stellar seismology - future students of stellar astrophysics will be expected to be conversant with the subject.

These are minor criticisms of an otherwise thoroughly good book. An instructor could choose material from the text to highlight certain areas, and as a whole it forms a sound beginner's course for those wishing to specialize in stellar astrophysics.

John Lattanzio is in the Institute of Geophysics and Planetary Physics, Lawrence Livermore National Laboratory, PO Box 808, Livermore, California 94550, USA.

entries (generally the better half) were part of an earlier Encyclopedia of Physical Science and Engineering. They can be identified because they were written around 1985 and contain no reference to anything later than that. The rest of the entries have been, well, cobbled together to try to give some kind of broader coverage of astronomy. So instead of getting an up-to-date survey of the whole of the subject by specialists, we have reprints of good but not very recent articles on some topics, together with more recent, but not very inspiring, articles on others (I except the entries dealing with Supernova 1987A here).

I am no expert on many of the subjects covered in the book, and (like most encyclopaedia users) have dipped into it here and there rather than attempting to read it from cover to cover. So I cannot vouch for its overall accuracy or otherwise. Still, Arno Penzias and Robert Wilson will be amused to find themselves described on p.174 as "engineers" who "detected a microwave background coming from all directions in space into a communications antenna they had designed and built for Bell Laboratories". American chauvinism enters into the section on space infrared facilities (p.9), where the US-Dutch-British IRAS satellite is described as the "highly successful NASA IR Astronomical Satellite"; likewise, in a list of future missions, the US SIRTF mission (which is yet to be approved and funded) is described but the European Space Agency's Infrared Space Observatory (ISO, which is now being built and is due for launch in 1993) is not mentioned.

I expect I will find myself using this encyclopaedia; there is a lot of good material here. But it is a pity that the publisher and editors didn't approach their task with a bit more integrity and give us a compilation of more uniform quality.

Michael Rowan-Robinson is in the School of Mathematical Sciences, Queen Mary College, Mile End Road, London E1 4NS, UK. 